\title{
BMJ Global Health ASHA Kirana: when digital technology empowered front-line health workers
}

\author{
V Srinidhi (D) , ${ }^{1}$ Baneen Karachiwala (D) , ${ }^{1}$ Aditi lyer (D) , ${ }^{1}$ Bhavya Reddy (D) , \\ Vinalini Mathrani, ${ }^{2}$ Neha Madhiwalla (D) ${ }^{3}$ Vani Periodi, ${ }^{4}$ Anuradha Sreevathsa, ${ }^{5}$ \\ Lakshmi Viswanatha (D) , ${ }^{6}$ Gita Sen
}

To cite: Srinidhi V, Karachiwala B, lyer A, et al. ASHA Kirana: when digital technology empowered front-line health workers. BMJ Global Health 2021;6:e005039. doi:10.1136/ bmjgh-2021-005039

Handling editor Seye Abimbola

Received 17 January 2021 Accepted 12 May 2021

Check for updates

(c) Author(s) (or their employer(s)) 2021. Re-use permitted under CC BY-NC. No commercial re-use. See rights and permissions. Published by BMJ.

${ }^{1}$ Ramalingaswami Centre on Equity and Social Determinants of Health, Public Health

Foundation of India, Bengaluru, Karnataka, India

${ }^{2}$ Independent Research Consultant, Bengaluru,

Karnataka, India

${ }^{3}$ ARMMAN, Mumbai,

Maharashtra, India

${ }^{4}$ Hitha Resource Unit, Mangaluru, Karnataka, India ${ }^{5}$ Swasti Health Catalyst, Bengaluru, Karnataka, India ${ }^{6}$ Aastrika, Bengaluru, Karnataka, India

Correspondence to

DrV Srinidhi; srinidhi.v@phfi.org

\section{ABSTRACT}

This practice paper describes our experience of implementing accredited social health activists (ASHA) Kirana, a digital technology-enabled Maternal Clinical Assessment Tool (M-CAT) and how the ASHAs felt empowered in the process. M-CAT aimed to train ASHAs to collect data that assists doctors in identifying maternal risks, in Karnataka, India. Systematic clinical assessment is not common in rural public health institutions. High caseloads, a tendency to 'normalise' maternal risks, varied competence of doctors and task shifting to insufficiently trained cadres may be some contributing factors. M-CAT was a response to this challenge. ASHAs asked a set symptom-cluster-based questions during home visits that were analysed by software algorithms to generate reports for doctors. M-CAT was implemented in one primary health centre with a group of 14 ASHAs, 2 auxiliary nurse midwives and 349 pregnant and postpartum women over 4 months. Our team worked with the ASHAs to refine the tool and supported them with training, hands-on assistance and regular debrief meetings. By learning how to collect individual-level data that they could interpret and act on, the ASHAs felt empowered with new knowledge on maternal risks. Their perfunctory data collection at home visits changed to substantive interactions with women and families, during which they captured pertinent qualitative information. The information asymmetry between doctors and ASHAs reduced. ASHAs started taking proactive steps on early indications of maternal risks. They changed from being mere transmitters of information to active users of it. Thus, technology-driven initiatives that include empowerment as an objective can strengthen the role of front-line workers in health systems.

\section{INTRODUCTION}

Accredited social health activists (ASHA) Kirana-literally, a 'ray of light for the ASHAs'-is how a group of Kannadaspeaking ASHAs described our Maternal Clinical Assessment Tool (M-CAT), a digital technology initiative in Karnataka, India. ASHAs are front-line workers in the Indian public health system. Our objective was to leverage digital technology to improve the quality of clinical assessments for every woman during antepartum and postpartum visits at primary

\section{Summary box}

Well-trained front-line health workers are critical if pregnancy-related risks are to be addressed promptly, especially when women and their families treat these as normal, and antenatal clinics are too crowded for doctors to pay individual attention to every woman.

- Clinical assessment tools powered by digital technologies provide an opportunity to empower frontline workers such as accredited social health activists (ASHAs).

- This practice paper, based on the experience of implementing a digital technology initiative, describes how strategies such as well-crafted training, followup field support and supportive debriefing meetings helped in empowering the ASHAs.

- Digital technology-enabled actionable data at the individual level can improve knowledge of maternal risk among front-line health workers and their communities, and prompt them to seek healthcare early.

health centres (PHCs), by training ASHAs to collect clinically meaningful data, and thereby assist medical officers (MOs).

Many mHealth interventions have focused mainly on improving health system factors at a population level, with little emphasis on individual-level data. These include tracking service delivery, providing automated educational information, improving health-seeking behaviour in communities, and building an accountability mechanism. ${ }^{1-4}$ Interventions like M-CAT that collect individual-level clinical data can respond better to the health needs of community members. ${ }^{5}{ }^{6}$ However, such interventions in obstetric care have been limited. ${ }^{78}$

Beyond the focus on individual-level data, we were keen to involve the ASHAs in a mutually beneficial way. Our early interactions with the ASHAs revealed that they were keen to understand and analyse data to avoid becoming mere data collectors. Too many mHealth initiatives use front-line workers 
to help technology achieve its potential. ${ }^{19} 10$ Evidence of technology serving to empower front-line workers by strengthening their abilities to interpret information and act is limited.

A few studies do point to the empowering potential of mHealth initiatives, but definitions of empowerment vary widely. A project in India that streamlined the job of data collection and set up automated alerts for high-risk cases made auxiliary nurse midwives (ANMs) feel supported. The authors termed this 'psychological empowerment'. ${ }^{11}$ In Ethiopia, the very act of owning a handheld device with unrestricted access to information made community health workers feel important. ${ }^{12}$ Interventions, such as Motech with automated health messages to raise awareness, are also believed to empower pregnant women. ${ }^{13}$

Our team at the Ramalingaswami Centre on Equity and Social Determinants of Health, Public Health Foundation of India (PHFI) strongly believed that while technology undoubtedly has its benefits, it should not reduce the significance of front-line workers if the health system is to retain its connection with people. M-CAT was built on this belief. It did not, per se, aim to empower the ASHAs. Our attempt was to provide ASHAs with new knowledge to help them collect and interpret data correctly and thereby support the MO, who clinically manages the patients at the PHC. However, by treating ASHAs as active partners with whom we refined and implemented the tool, we ended up empowering them. For the purposes of this paper, we frame empowerment as a process that goes beyond capacity building to build confidence and a sense of self-worth. ${ }^{14}$

In this paper, we focus on this positive impact on ASHAs at the end of a 4-month long feasibility study in 2017, during which we implemented and evaluated the M-CAT. Our study in a poor rural region was supported by the National Health Systems Resource Centre, New Delhi and the National Health Mission, Karnataka. It evaluated acceptability of the tool with stakeholders and the processes involved in rolling it out.

\section{M-CAT: THE TOOL AND THE FEASIBILITY STUDY Why strengthen clinical assessments during pregnancy?}

Our implementation research on maternal safety in Koppala district, Karnataka, ${ }^{15-17}$ identified four interconnected contributors to the problem of poor clinical care for rural women during the pregnancy.

First, a tendency among healthcare providers, as well as women and their families, to 'normalise' commonly encountered risks. For example, in poor communities where anaemia is highly prevalent, doctors tend not to actively manage diminished haemoglobin levels until they fall perilously.

Second, time constrained doctor-patient interactions due to large inflows of women for antepartum care and delivery, especially after the roll-out of the National Rural Health Mission. In such a scenario, women often fail to receive individual clinical attention. Harried doctors tend to fall back on a checklist of discrete symptoms to quickly screen women instead of using clusters of symptoms, signs and test results to guide their diagnoses of maternal risks and comorbidities. The latter is essential if clinical diagnoses are to be rigorous. For example, we found that border-line blood pressure did not prompt doctors to look for other indications of pregnancy-induced hypertension, such as swelling of the legs and albumin in urine.

Third, inconsistent clinical capacity among doctors (some being non-allopaths), due to variations in the content and quality of their basic training, and of their clinical exposure during internships. Inconsistencies also arise due to lax implementation of standard protocols.

Fourth, task shifting from higher to lower levels of care, often with inadequate supportive supervision. For example, although ANMs are formally responsible for tracking 'risk cases' identified by the MO, the ASHAs are often the ones who actually follow up. With insufficient training and only sporadic supervision by the ANMs, ASHAs provide little more than nutrition-linked information to women. They are also expected to collect data about women's antenatal well-being. Yet, without sufficient knowledge, the ASHAs tend to ask non-specific questions ('do you have any problems?'), and report symptoms that are neither graded for severity nor linked to action. Such task shifting is counterproductive. ASHAs invest considerable time and effort in collecting information that is not particularly useful for the MO, who requires reliable, actionable information.

\section{M-CAT: a digital tool for effective risk identification and clinical management}

As an information technology-enabled tool, M-CAT was designed to enable ASHAs to collect clinical data from pregnant and postpartum women and assist MOs to identify maternal risks. It expanded the current focus on age, height, parity and spacing by including information about a woman's obstetric and medical history, as well as commonly occurring comorbidities. More importantly, it emphasised a cluster-based approach to diagnosing and grading the severity of both maternal risks and comorbidities. It oriented ASHAs to the idea that maternal risks require prompt action and provided doctors with casesheets functioning like protocols to prompt systematic clinical assessments. In its design, the tool was an individualised response mechanism to clinical needs rather than a way of aggregating population level data to guide health programme planning.

M-CAT has four major components (table 1).

Drawing from a handbook on maternal risks, ${ }^{18} 19$ the forms included all potentially life-threatening obstetric conditions and comorbidities (acute and chronic) that can be algorithmically deduced from clusters of symptoms/signs and simple tests. Our medical team together with a group of experienced, practising obstetricians finalised this list of conditions (table 2).

The tool excluded health problems that can be diagnosed only after detailed investigations (eg, domestic 
Table 1 Components of M-CAT

\begin{tabular}{ll}
\hline Component & Description \\
\hline Forms & $\begin{array}{l}\text { A set of paper forms containing questions to systematically collect information on symptoms, signs } \\
\text { and test results of risk conditions during pregnancy and the postpartum period. The forms are to be } \\
\text { administered by the ASHAs during their home visits }\end{array}$ \\
Software & $\begin{array}{l}\text { A multilingual software application built on diagnostic algorithms, that generates rigorous initial clinical } \\
\text { diagnoses, based on the information collected by the ASHAs. The algorithms consider clusters of } \\
\text { symptoms, signs and test results and do not rely on discrete non-specific symptoms. For example, a } \\
\text { woman with swelling of the limbs, tiredness and high blood pressure will be provisionally identified with } \\
\text { both anaemia and pre-eclampsia }\end{array}$ \\
\hline Reports & $\begin{array}{l}\text { The reports generated by the software include (1) summary reports for the ASHAs, ANMs and the MO, (2) } \\
\text { one medical case sheet per woman for the MO and (3) a set of worksheets for the researcher }\end{array}$ \\
Communication & $\begin{array}{l}\text { The reports are communicated to the ASHAs, ANMs and MO, prompting them to take timely action to } \\
\text { manage risks }\end{array}$ \\
\hline
\end{tabular}

ANMs, auxiliary nurse midwives; ASHAs, accredited social health activists; M-CAT, Maternal Clinical Assessment Tool; MO, medical officers.

violence, postpartum depression) or sophisticated tests (eg, cortical venous thrombosis). It also excluded diseases that must be kept confidential (eg, HIV).

Questions in the tool were arranged in a systematic way. Algorithms to provide preliminary clinical assessments were created using the clinical guidelines in our handbook. The software was developed as an offline application written in Java, running on a Tomcat server with a MySQL database. The software had a simple user interface and could generate reports for each woman, identifying any risks and their severity, in a format similar to a short clinical case sheet.

\section{The implementation and feasibility study}

The feasibility study was conducted in all villages under one PHC in Koppala district, Karnataka. M-CAT implementation involved 14 ASHAs, 2 ANMs and the PHC MO, as well as two project functionaries (data entry operator (DEO) and link worker (LW)). A total of 349 pregnant and postpartum women were registered and followed up over four successive 30-day cycles between September 2016 and January 2017. Our team monitored and evaluated this implementation closely, via direct observation of ASHA's home visits, participant observation of debrief meetings, focus group discussions with ASHAs, in-depth interviews with randomly selected women and their families, and review of the clinical database generated through M-CAT work cycles. While monitoring the roll out, the research and implementation teams met regularly to share and analyse field experiences.
A detailed discussion on the methods and findings from the feasibility study are beyond the scope of this paper. Our focus is to describe our experience of developing and implementing the M-CAT by working closely with the ASHAs. We reflect on the strategies that we employed in working with the ASHAs and how the ASHAs felt empowered as a result of those strategies.

\section{ASHAS AS ACTIVE PARTNERS}

Our collaboration with the ASHAs involved learning from them about the contextual challenges of their work, which helped us fine-tune the M-CAT (including questions in the forms and the work cycles), and building their knowledge and skills.

\section{Refining the forms}

Our first interaction with the ASHAs focused on refining the clinical questions that were already phrased using simple yet accurate language. These were tweaked further through dialogue between the ASHAs and our medical team: the former suggesting modifications to the questions in the form, the latter vetting these for accuracy. The aim was to have questions that can be administered easily while keeping the women comfortable (eg, avoiding sensitive questions requiring privacy).

\section{Building knowledge and skills}

We then held a 3-day training workshop for 14 ASHAs in the PHC along with two ANMs. Employing interactive

Table 2 Conditions for which questions were included in the form

\section{Type of health conditions Specific conditions considered in the form}

Obstetric conditions

Acute comorbidities
Miscarriage, ectopic pregnancy, pregnancy induced hypertension, antepartum haemorrhage, intrauterine death, premature rupture of membranes, postpartum haemorrhage, sepsis, postpartum psychosis, mastitis and breast abscess

Pulmonary tuberculosis, malaria, urinary tract infection, reproductive tract infections (as a generic group)

Chronic comorbidities
Anaemia, diabetes, chronic hypertension, heart disease, thyroid dysfunction 
methodologies, the training aimed to enthuse and orient each functionary to the forms, processes and their roles. Half a day was also spent on orienting the MO, LW and DEO to their respective roles.

Following this initial training, the ASHAs, LW and DEO were provided practical field-based training and support. The research team and LW accompanied the ASHAs over 2 days for hands-on training in form filling. This activity provided a baseline picture of the ASHAs' capabilities and indicated the type of support they would need. It also initiated the LW into her role of providing hands-on support.

Six of the 14 ASHAs who were less literate and communicative, needed more support than the others. Continuous handholding was crucial for the first couple of cycles as these six ASHAs were failing to adhere to instructions that were critical for eliciting symptoms. The other eight ASHAs required much less oversight to ensure that formfilling proceeded as mandated.

Over time, even the six ASHAs who required extensive support became adept at form-filling. Sheer practice also helped them elicit symptoms expertly. They were given an incentive of Rs50 per form to fill it without errors, which may have sustained their motivation to learn and to be accurate.

By the end of the fourth cycle, all ASHAs had become very familiar with the questions, which they articulated clearly, and rarely made errors in filling the forms.

Complementing the individual support mechanisms were monthly debrief meetings at the PHC. The ASHAs and project staff (LW, DEO) as well as representatives of our implementation and monitoring \& evaluation teams attended these meetings that aimed to: (1) give ASHAs feedback on their performance; (2) allow them to talk about their experiences, challenges and concerns; (3) troubleshoot, clarify queries and retrain them (as required) and (4) develop action plans for women at risk (identified through M-CAT or otherwise). The quality of the interactions at the debrief meetings was usually very good, with the ASHAs participating actively and demonstrating their openness to learning

\section{Dovetailing with the existing structure}

We learnt from our field interactions that only two cadres of health workers effectively engage with pregnant and postpartum women: the ASHAs and the doctors. The role of the ANMs had significantly reduced since the introduction of the ASHAs, and the staff nurses merely supported the doctors. Given the serious time constraints faced by the doctors, the M-CAT had to be designed with the ASHAs in mind.

Since the ASHAs were already saddled with multiple responsibilities, the M-CAT had to dovetail with their existing responsibilities. Moreover, it was important for this activity to improve the efficiency of the doctor without becoming onerous.

Keeping this in mind, we integrated data collection into the ASHAs' schedule of activities during regular home visits. We also ensured that M-CAT forms systematised and improved the questions that the ASHAs would ask anyway. In addition, we asked the ASHAs to plan their home visits before the scheduled monthly ANC clinics at the PHC, leaving enough time for the data to be processed and for reports to be generated for the MO.

\section{HOW M-CAT EMPOWERED THE ASHAS}

The ASHAs were initially apprehensive about their ability to play the role envisaged for them, due to their lack of experience and low literacy. They were also unsure about how women and their families would respond to the forms, because of the popular belief that surveys only benefit external organisations. While the ASHAs did acknowledge that M-CAT linked visits and responsibilities aligned with their existing schedules and roles, they were also concerned that the additional time required for home visits would prove burdensome. Despite this, they could see that M-CAT had the potential to add value to their work.

By the end of the fourth month, the ASHAs reported that the M-CAT had transformed their interactions with women and families. They felt that prior to M-CAT, their visits were unstructured, and gave only a general sense of the women's health status. But, with M-CAT the ASHAs were trained to collect data with an understanding of what each piece of information meant. They believed that the very act of eliciting information about symptoms of risk enabled them to have more substantive interactions with women.

Regular form filling on specific symptoms had two important consequences. It made women and their families more aware of risk symptoms and helped mitigate the tendency to treat these as normal. As for the ASHAs, repeated use of the forms reinforced their training and provided them with an opportunity to learn something new, while giving them the confidence to ride on their freshly acquired skills.

While their regular training had already taught them to elicit symptoms, M-CAT helped them become more knowledgeable and discerning about maternal risks and showed them how to recognise simple clinical signs. By the end of the fourth month, they understood that specific symptoms (eg, severe bleeding, pale conjunctiva) connoted risk as opposed to minor symptoms such as mild occasional headaches or constipation. They realised the significance of specific symptom qualifiers (eg, severe headache; foul smelling white discharge). They learnt that the location of symptoms is important (eg, pain in the 'upper part' of the abdomen). They also developed the ability to recognise fine distinctions between symptoms (eg, dizziness vs unconsciousness) and to link symptoms with risk conditions.

Equally noteworthy, ASHAs often recorded qualitative information that they deemed important along the margins of the form (eg, 'lack of interest in feeding the baby', 'feels weak'). This speaks to the seriousness and 
interest with which they elicited information, and their skill to find 'space' to tell their story. It also points to the need to design data collection tools to capture information in a format that is in sync with how front-line workers like ASHAs communicate.

Remarkably, the ASHAs additionally felt that greater familiarity with risk conditions had implications for their status within the community. They reported that families were now reaching out to them for support-a dramatic turnaround from a time when they had to repeatedly urge women and families to use healthcare services. There was also evidence of the ASHAs as a group and some families proactively responding to risks. Sometimes, risks were picked up and addressed outside of scheduled PHC visits before symptoms escalated into full-blown emergencies.

Overall, our attempt to enhance the ASHAs' existing knowledge and skills made them feel empowered by knowing more, by being able to interpret information and taking action based on it. They distinctly felt a shift from being mere conveyors to active users of information. The ASHAs also felt that their emergent familiarity with maternal risk made it easier for them to discuss the health of pregnant/postpartum women under their jurisdiction with the MO. In doing so, they had begun to chip away at the information asymmetry that underpinned their subordination in the public health system.

\section{LIMITATIONS AND CHALLENGES}

While the empowering effect of M-CAT was striking, the tool itself was preliminary in nature, paper-based, and faced challenges in real-time diagnosis and tracking. For example, while data on symptoms and signs pertained to the current month, laboratory test results were usually carried over from previous months, Thus an accurate current diagnosis was not possible in some cases. Additionally, when ASHAs and/or women went directly to a higher centre outside our implementation geography, the information was not communicated to the PHC MO. A scaled-up version of the M-CAT with handhelds spreading across geographies can effectively tackle these limitations.

\section{CONCLUSION}

ASHAs contribute in important ways to strengthening the healthcare system in rural India. They engage with communities and facilitate their access to health service providers. Our experience with M-CAT revealed that digital technology can potentially be a 'ray of light' for the front-line workers like ASHAs. ASHAs with their new knowledge were able to make women and their families aware about maternal risks, prompting them to recognise symptoms and take action quickly when required. Therefore, empowering front-line workers like ASHAs with knowledge and actively supporting their inherent skill to engage with the community should be important strategic objectives of digital technology interventions.
Acknowledgements The authors would like to thank project-appointed staff for their valuable contributions: Mr Narasimha Raj Kammar (the DEO) and Ms Phakeeramma Budakunti (the LW).

Contributors VS and BK reviewed the literature, wrote the first draft of this article and revised it following peer review. Al, BR and VM reviewed and made substantive revisions to all drafts. NHM reviewed an early draft of the paper and helped in reviewing the literature. VP and LV trained the participants. VS and AS developed the clinical content of the tool, including the clinical algorithms. VM and BK implemented the tool in the field, and monitored the study. BR and NHM did the evaluation. GS and Al conceived of the study, and mentored the team. All authors read and approved the final manuscript.

Funding This study was supported by Bill \& Melinda Gates Foundation.

Competing interests None declared.

Patient consent for publication Not applicable.

Ethics approval The study was approved by PHFl's ethics committee.

Provenance and peer review Not commissioned; externally peer reviewed.

Data availability statement Deidentified summary of qualitative data from interviews and focus groups discussions are available from the correspoding author upon reasonable request.

Open access This is an open access article distributed in accordance with the Creative Commons Attribution Non Commercial (CC BY-NC 4.0) license, which permits others to distribute, remix, adapt, build upon this work non-commercially, and license their derivative works on different terms, provided the original work is properly cited, appropriate credit is given, any changes made indicated, and the use is non-commercial. See: http://creativecommons.org/licenses/by-nc/4.0/.

\section{ORCID iDs}

V Srinidhi http://orcid.org/0000-0002-5569-3719

Baneen Karachiwala http://orcid.org/0000-0002-2994-4297

Aditi lyer http://orcid.org/0000-0002-2177-0755

Bhavya Reddy http://orcid.org/0000-0002-1281-2810

Neha Madhiwalla http://orcid.org/0000-0002-0922-7186

Lakshmi Viswanatha http://orcid.org/0000-0003-4508-2217

Gita Sen http://orcid.org/0000-0001-9010-074X

\section{REFERENCES}

1 Aranda-Jan CB, Mohutsiwa-Dibe N, Loukanova S. Systematic review on what works, what does not work and why of implementation of mobile health (mHealth) projects in Africa. BMC Public Health 2014;14:188.

2 Ahsan A, Raihan A. Understanding mHealth impact among Aponjon (MAMA Bangladesh) subscribers through a phone survey in Bangladesh. In: Marsden G, May J, Chetty M, et al, eds. ICTD '13 Proceedings of the Sixth International Conference on Information and Communications Technologies and Development: Notes-Volume 2. Association for Computing Machinery, 2013: 1-4.

3 LeFevre AE, Mohan D, Hutchful D, et al. Mobile technology for community health in Ghana: what happens when technical functionality threatens the effectiveness of digital health programs? BMC Med Inform Decis Mak 2017;17:27.

4 Barron P, Peter J, LeFevre AE, et al. Mobile health messaging service and helpdesk for South African mothers (MomConnect): history, successes and challenges. BMJ Glob Health 2018;3:e000559.

5 Adepoju I-OO, Albersen BJA, De Brouwere V, et al. mHealth for clinical decision-making in sub-Saharan Africa: a scoping review. JMIR Mhealth Uhealth 2017;5:e38.

6 Peiris D, Praveen D, Johnson C, et al. Use of mHealth systems and tools for non-communicable diseases in low- and middleincome countries: a systematic review. J Cardiovasc Trans/ Res 2014;7:677-91.

7 Modi D, Desai S, Dave K, et al. Cluster randomized trial of a mHealth intervention "ImTeCHO" to improve delivery of proven maternal, neonatal, and child care interventions through community-based Accredited Social Health Activists (ASHAs) by enhancing their motivation and strengthening supervision in tribal areas of Gujarat, India: study protocol for a randomized controlled trial. Trials 2017; 18:270.

8 Shah P, Madhiwala N, Shah S, et al. High uptake of an innovative mobile phone application among community health workers in rural India: an implementation study. Natl Med J India 2019;32:262-9. 
9 PHilbriCk WC. mHealth and $\mathrm{MNCH}$ : state of the evidence. trends, gaps, stakeholder needs, and opportunities for future research on the use of mobile technology to improve maternal, newborn, and child health. Washington; 2013. https://www.gfmer.ch/mhealth/ coursefiles2013/mhealthmnch-evidence-final.pdf

10 Sondaal SFV, Browne JL, Amoakoh-Coleman M, et al. Assessing the effect of mHealth interventions in improving maternal and neonata care in low- and middle-income countries: a systematic review. PLoS One 2016;11:e0154664.

11 Pandey P, Power ZY. Technology and Empowerment. In: Bandi RK $\mathrm{CR} \mathrm{R}$, Klein S, et al, eds. IFIP joint working conference on the future of digital work: the challenge of inequality. Springer, 2020: 165-79.

12 Little A, Medhanyie A, Yebyo $\mathrm{H}$, et al. Meeting community health worker needs for maternal health care service delivery using appropriate mobile technologies in Ethiopia. PLoS One 2013;8:e77563.

13 Al Dahdah M. Health at her fingertips: development, gender and empowering mobile technologies. Gend Technol Dev 2017;21:135-51.

14 Zoabi K, Gal I. Chapter 4: Empowerment Potential of Social Work Techniques among Practitioners in Israel and the USA. In: Nikku B, ed. Global social work: cutting edge issues and critical reflections. Thompson Rivers University, 2020.

15 Iyer A, Sen G, Sreevathsa A, et al. Verbal autopsies of maternal deaths in Koppal, Karnataka: lessons from the grave. BMC Proc 2012;6:P2.

16 Shankar M, Srinidhi V. Deconstructing clinical knowledge of obstetric care at the primary level: insights from rural Karnataka, India. Paper presented at: Social Science and Medicine Conference Health Systems in Asia 2013: Equity, Governance and Social Impact; December 2013, National University of Singapore, Singapore, 2013.

17 Godt S, Agyepong I, Flores W, Sen G. Healthy lives for vulnerable women and children. applying health systems research. Ottawa International Development Research Centre; 2017. www.idrc.ca/ healthy-lives

18 Gender and health equity project. identifying and addressing maternal risks: a Handbook for healthcare providers. Indian Institute of Management Bangalore 2015.

19 lyer A, Srinidhi V, Sreevathsa A, et al. Adapting maternal health practice to co-morbidities and social inequality: a systematic approach. Can J Public Health 2017;108:448-51. 